\title{
Management of External Cervical Root Resorption in Mandibular Molar
}

\author{
${ }^{1}$ Mahendran Kavitha, ${ }^{2}$ Shekar Shobana
}

\begin{abstract}
External root resorption of teeth takes its origin from periodontal tissues but is often an endodontic enigma. A combined endoperio management is required if the external resorption involves both entities. This article describes the management of an external resorption in mandibular first molar by intentional reimplantation using cone-beam computed tomography (CBCT) as an adjunctive diagnostic aid. Following atraumatic extraction of 36 , endodontic treatment was performed extraorally, followed by sealing of the resorptive site using Biodentine. The periodontal procedures included guided tissue regeneration using a bioresorbable membrane and placement of osseograft for bone regeneration. After a follow-up period of 10 months, the patient was totally asymptomatic. Intentional reimplantation is a viable treatment option for external root resorption of inaccessible sites.
\end{abstract}

Keywords: Biodentine, External cervical root resorption, Guided tissue regeneration, Intentional reimplantation.

How to cite this article: Kavitha M, Shobana S. Management of External Cervical Root Resorption in Mandibular Molar. J Oper Dent Endod 2016;1(1):39-45.

Source of support: Nil

Conflict of interest: None

\section{INTRODUCTION}

External cervical root resorption is an idiopathic localized destructive lesion of the root that could be asymptomatic and frequently confronted as an incidental diagnosis in the dental office. Resorption can be defined as 'a condition associated with either a physiologic or a pathologic process resulting in the loss of dentin, cementum or bone'. ${ }^{1}$ Andreasen has classified tooth resorption into two broad categories namely-internal and external. Internal resorption includes inflammatory and replacement resorption, external resorption includes surface resorption, external inflammatory resorption, external replacement resorption, external cervical resorption (ECR), transient apical breakdown. ${ }^{2,3}$

${ }^{1}$ Professor and Head, ${ }^{2}$ Postgraduate Student

1,2Department of Conservative Dentistry and Endodontics, Tamil Nadu Government Dental College and Hospital, Chennai, Tamil Nadu, India

Corresponding Author: Mahendran Kavitha, Professor and Head, Department of Conservative Dentistry and Endodontics Tamil Nadu Government Dental College and Hospital, Chennai Tamil Nadu, India, e-mail: kavithaendo@yahoo.com
External cervical resorption also known as external cervical invasive resorption (ECIR) is one form of resorption that has been vaguely explained. It has been defined as 'a localized, periodontally derived, inflammatory tissue loss that begins on the root surface, at or below its epithelial attachment'. ${ }^{4}$ Heithersay ${ }^{5}$ has classified ECIR into four types: Class 1 denotes a small cervical resorption with shallow dentin penetration and, usually, a soft-tissue defect that bleeds upon probing. Radiographically, it may appear as a small coronal radiolucency, class 2 indicates a well-defined invasive resorptive lesion that has infiltrated close to the coronal pulp but they do not extend into radicular dentin. Class 3 denotes a deeper invasion of dentin by resorbing tissue, not only involving the coronal dentin but also extending into the coronal third of the root. The radiographic appearance in class 3 lesions will often appear moth-eaten, with small, finger-like projections, and a radiopaque line separates the resorption from the root canal. Class 4 indicates a large invasive resorptive process that has extended beyond the coronal third of the root.

The various treatment modalities for ECIR includes no treatment, surgical and nonsurgical approaches and lastly extraction should also be considered as a possible treatment option. ${ }^{5}$ One of the surgical treatment modalities for ECIR is intentional reimplantation. Intentional reimplantation is defined by Grossmann as the intentional removal of a tooth and its reinsertion into the socket after endodontic manipulation or obturation of the canals or both. ${ }^{6}$ Whatever the treatment modality, the basic aim of treatment plan for ECIR remains the same: inactivation of the active resorbing tissue and reconstruction of the lost resorptive site using a biomaterial or membrane. ${ }^{5}$

The diagnosis of ECIR is a challenge as there is no characteristic clinical or radiographic presentation. Recently, three-dimensional (3D) digital imaging modalities have been used for diagnosis of resorption. Cone-beam computed tomography (CBCT) has found vast applications in the field of Conservative Dentistry and Endodontics particularly in resorption, it is an invaluable diagnostic aid in assessing the 3D orientation and extent of the resorptive lesion. ${ }^{7}$

The following case report describes the surgical management of a class 3 ECIR by intentional reimplantation using CBCT as a diagnostic adjunct. 


\section{CASE REPORT}

\section{Clinical Examination}

A 27-year-old male patient reported to the Department of Conservative Dentistry and Endodontics with the chief complaint of dull pain and swelling in the left lower back toot region for the past 2 days. Patient's medical and dental history were noncontributory. He had lost his 37 few years back to dental caries. Extraoral examination revealed mild facial asymmetry due to swelling in the lower left half of the face. Intraoral examination revealed fractured mesiolingual cusp in 36, intraoral swelling seen as obliteration of mucobuccal fold extending from mesial of 35 to distal aspect of 37 and severe calculus deposits. On probing, there was profuse bleeding from the mesial interdental region of 36 with a distinct scraping sound.

\section{Radiographic Examination}

Intraoral periapical radiographic examination revealed radiolucent lesion on the mesial aspect of the cervical third of the mesial root of 36 . The lesion had characteristic spiky finger-like projections with ragged irregular margins and there was crestal bone loss in relation to 36 (Fig. 1A). Correlating clinical and radiographic findings the case was provisionally diagnosed as external cervical root resorption in 36 . The differential diagnosis was subgingival caries and cervical burn out. As the radiographic findings were not supportive enough to confirm the diagnosis, CBCT (CS 9300, Carestream, Rochester, New York, USA) in limited field of view was done.

\section{CВCT Findings}

Cone-beam computed tomography evaluation of 36 revealed the presence of a $4 \times 3 \mathrm{~mm}$ resorptive lesion on the mesial aspect of 36 . The $3 \mathrm{D}$ extent of the lesion was precisely determined using the various axial images of $\mathrm{CBCT}$, and pulpal involvement was confirmed in the $\mathrm{CBCT}$ analysis (Figs $1 \mathrm{~B}$ to $\mathrm{F}$ ).

\section{Diagnosis}

The case was diagnosed as Heithersay class 3 ECIR.

\section{Treatment Plan}

The treatment plan is as follows:

- Oral prophylaxis and preliminary hematological investigations

- Atraumatic extraction of 36

- Extraoral endodontic treatment of 36 followed by apicoectomy, retrograde preparation and retrograde filling
- Curettage of the resorptive site followed by its repair with a biocompatible material

- Reimplantation of the tooth in its physiological position in the socket

- Curettage of the periodontal tissues and placement of a barrier membrane and bone graft to promote healing

\section{Treatment Procedure}

Preliminary hematological investigations were done followed by thorough oral prophylaxis. Under systemic antibiotic coverage and inferior alveolar nerve block with lignocaine $2 \%$ and 1:80,000 adrenaline (Lignox $2 \%$ A, Warren, Indoco Remedies), atraumatic extraction of 36 was done (Fig. 2A). The tooth was immediately stored in sterile physiological saline (Fig. 2B) until further use. The patient was asked to bite lightly on sterile cotton until subsequent procedures were carried out.

The curettage of the resorptive site was first carried out using spoon excavators (Fig. 3A) and irrigated with saline. Extraoral endodontic treatment was performed in extracted 36 by gaining access using sterile diamond points (TF 12, Mani Inc, Germany) and high speed airotor handpiece (NSK, Japan). Distal, mesiobuccal and mesiolingual canals were located and negotiated with patency file no. $10 \mathrm{~K}$-file (Mani Inc, Germany) followed by initial apical enlargement using no. $20 \mathrm{~K}$-file (Mani Inc, Germany). The biomechanical preparation of the canals were done with rotary ProTaper file no. F1 with intermittent saline irrigation. Sodium hypochlorite was avoided as it could be toxic to the periodontal ligament cells. Obturation was done with F1 size gutta-percha and zinc oxide eugenol sealer (Fig. 2D). This was followed by apicoectomy of the apical $3 \mathrm{~mm}$ of the roots, retrograde preparation and retrograde filling (Fig. 2C) with high strength posterior restorative glass Ionomer cement (Fuji IX, GC Corporation, Tokyo, Japan). Finally, the resorptive area was reconstituted with Biodentine (Fig. 3B) (Septodont, Saint Maur-des Fosses, France), a novel calcium trisilicate-based cement and access cavity temporized with IRM (Dentsply Caulk, Milford, USA).

During the entire span of the extraoral procedure, the tooth was gently grasped with a sterile gauze soaked in saline to retain the viability of the periodontal ligament cells. Just prior to reimplantation of the tooth the root surfaces were surface treated with tetracycline for 2 minutes. The extraoral procedures were completed within 27 minutes.

\section{Reimplantation}

Once the scheduled extraoral procedures were completed, the extraction socket was gently rinsed with 

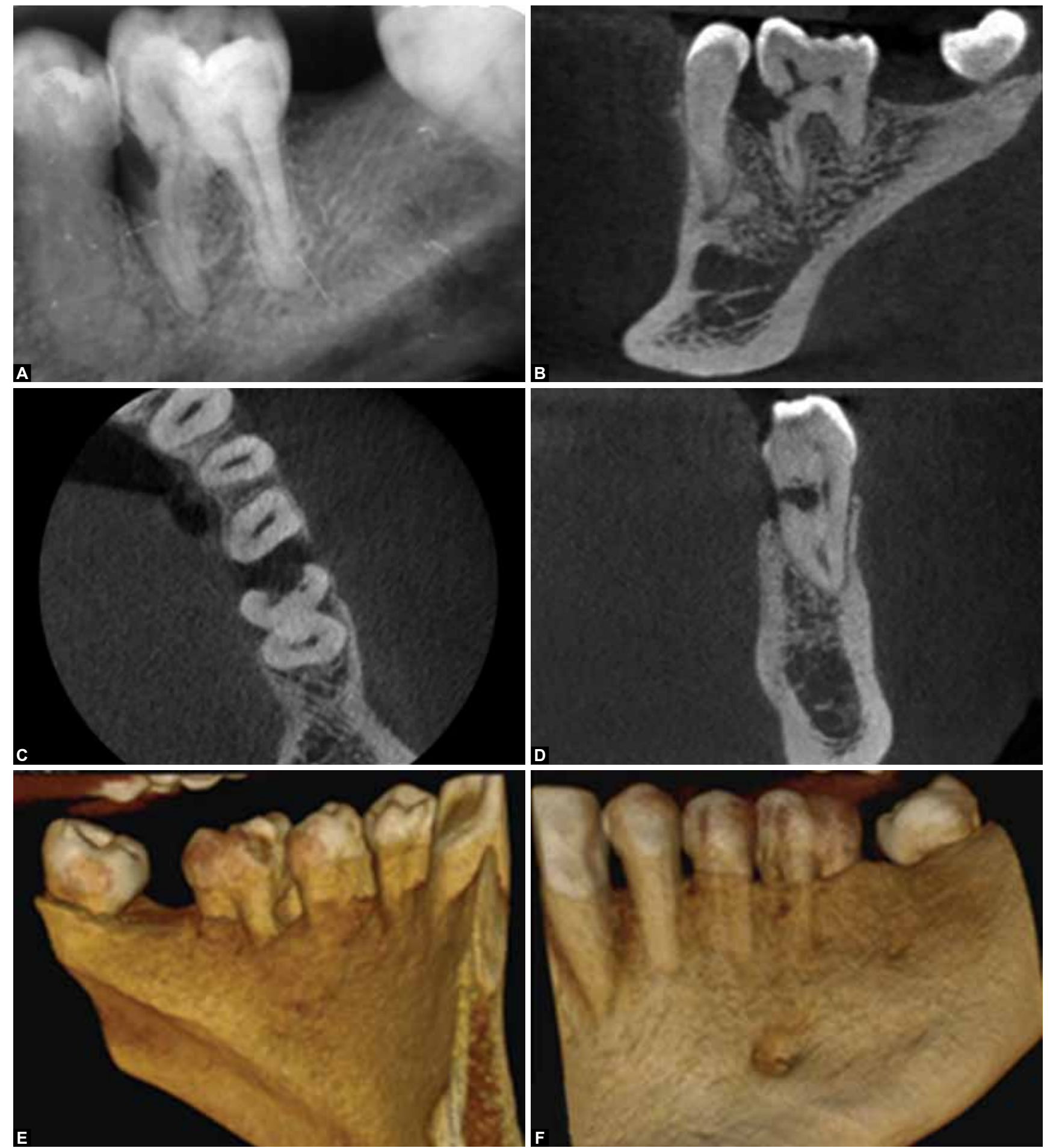

Figs 1A to F: (A) Preoperative radiograph of 36, (B) sagittal view of CBCT showing the resorptive site, (C) transverse view of CBCT showing the resorptive site, $(D)$ coronal view of $C B C T$ showing the resorptive site and (E and $F) 3 D$ reconstructed images of the $C B C T$ scan

saline to wash away the clot and reimplantation of the tooth into its socket was done by grasping the crown and positioning it into the socket after proper orientation (Fig. 3C). The patient was asked to bite on moist cotton firmly for few minutes.

\section{Periodontal Procedures}

After repositioning the tooth, the gingival tissues were curetted (Fig. 3D). A bioresorbable collagen barrier membrane (Healiguide, Advanced Biotech, India) was placed on the furcation area for guided tissue regeneration (Fig. 3E) followed by the placement of freeze-dried demineralized xenograft (Osseograft, Advanced Biotech, India) for osseous regeneration (Fig. 3F). The gingival tissues were stabilized using simple interrupted sutures and periodontal pack (Coe-Pak) was placed. Prior to the placement of the periodontal pack, an immediate postoperative radiograph was taken to ensure the 

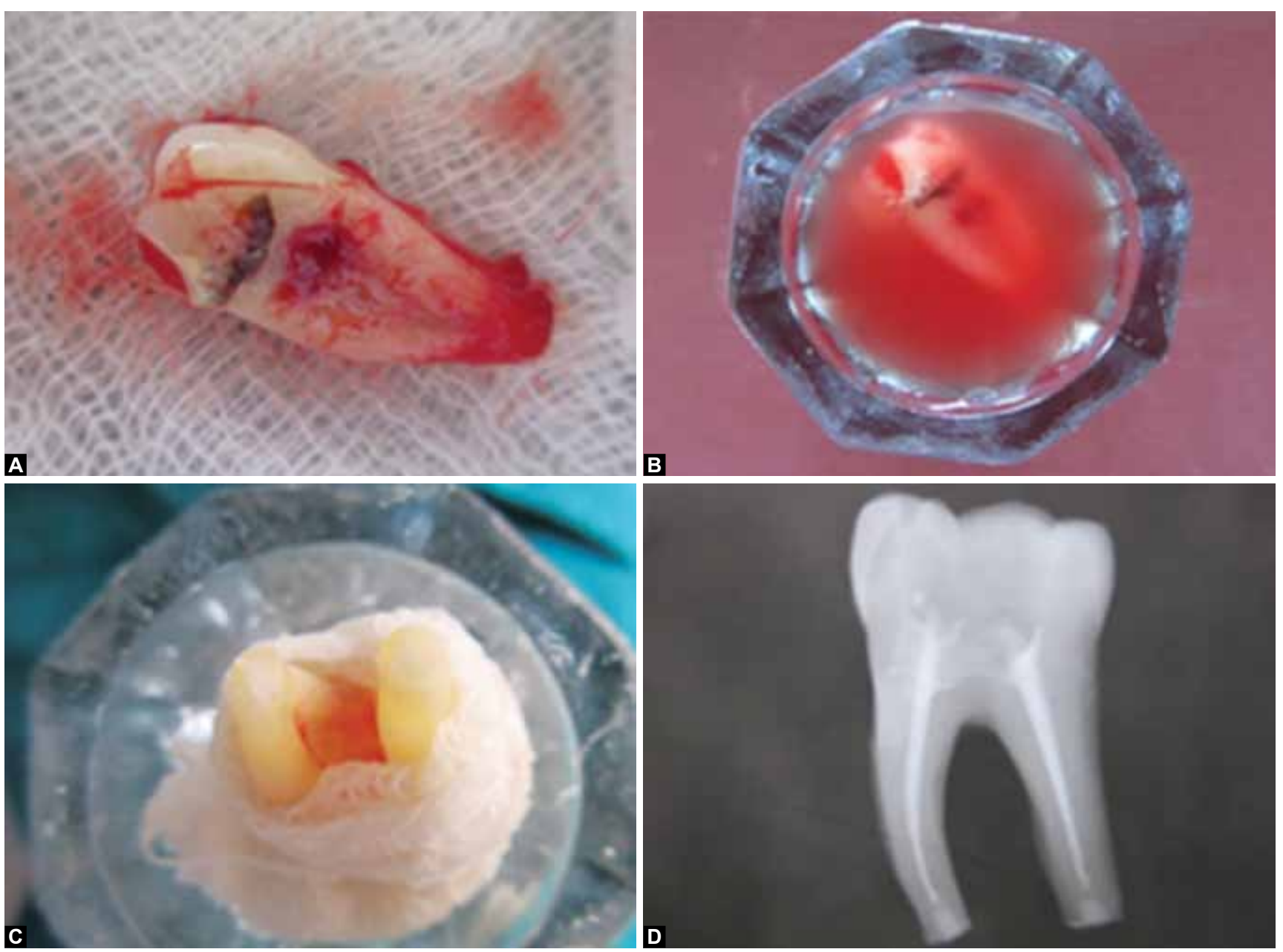

Figs 2A to D: (A) Atraumatic extraction, (B) storage in saline immediately after extraction, (C) extraoral endodontic treatment, apicoectomy and retrograde filling done and (D) extraoral radiograph showing endodontic treatment and retrograde filling

tooth position (Fig. 4A). The pack acts as soft flexible splint until definitive splinting procedure can be done. Tooth no. 36 was relieved from occlusion. Patient was advised to continue the prescribed antibiotics and antiinflammatory, prophylactic chlorhexidine mouth rinse was prescribed, and patient was advised to take soft diet for 3 days.

\section{Postoperative Follow-up}

The patient was reviewed after 24 hours and evaluated for the presence of bleeding, intactness of the sutures and periodontal pack. During the first week recall appointment, the periodontal pack was removed and splinting was done with light cure composite resin which was removed 2 weeks later. Access cavity was restored with light-cured composite restoration (Tetric N Ceram, Ivoclar Vivadent).

Periodic clinical (Fig. 4D) and radiographic evaluation was done at 6 and 10 months intervals (Figs $4 \mathrm{~B}$ and C). Radiograph revealed the absence of new resorptive lesion and satisfactory bony regeneration. There was no periapical pathology or clinical signs of tooth mobility.

\section{DISCUSSION}

External cervical root resorption is frequently an asymptomatic condition associated with the cervical region of the tooth wherein the source of clastic process originates from the cells of the periodontium. It may not be associated with any evident etiological factors and can progress rapidly with gross destruction of dentin. Some cases can have concomitant pulpal involvement and may require endodontic therapy as well. Whenever there is simultaneous pulpal involvement, the treatment of the affected tooth should be aimed at treating the diseased pulpal tissue and the periodontium. In cases where there is limited intraoral access, intentional reimplantation is considered as a viable option for the management of ECIR.

Diagnosis of cervical root resorption can be done clinically, radiographically and recently 3D imaging modalities are available for exact localization of the lesion. Clinical and radiographic diagnosis is established by nonspecific and vague findings. Ahmed et $\mathrm{al}^{8}$ in 2014 have published a case report in which they have established 

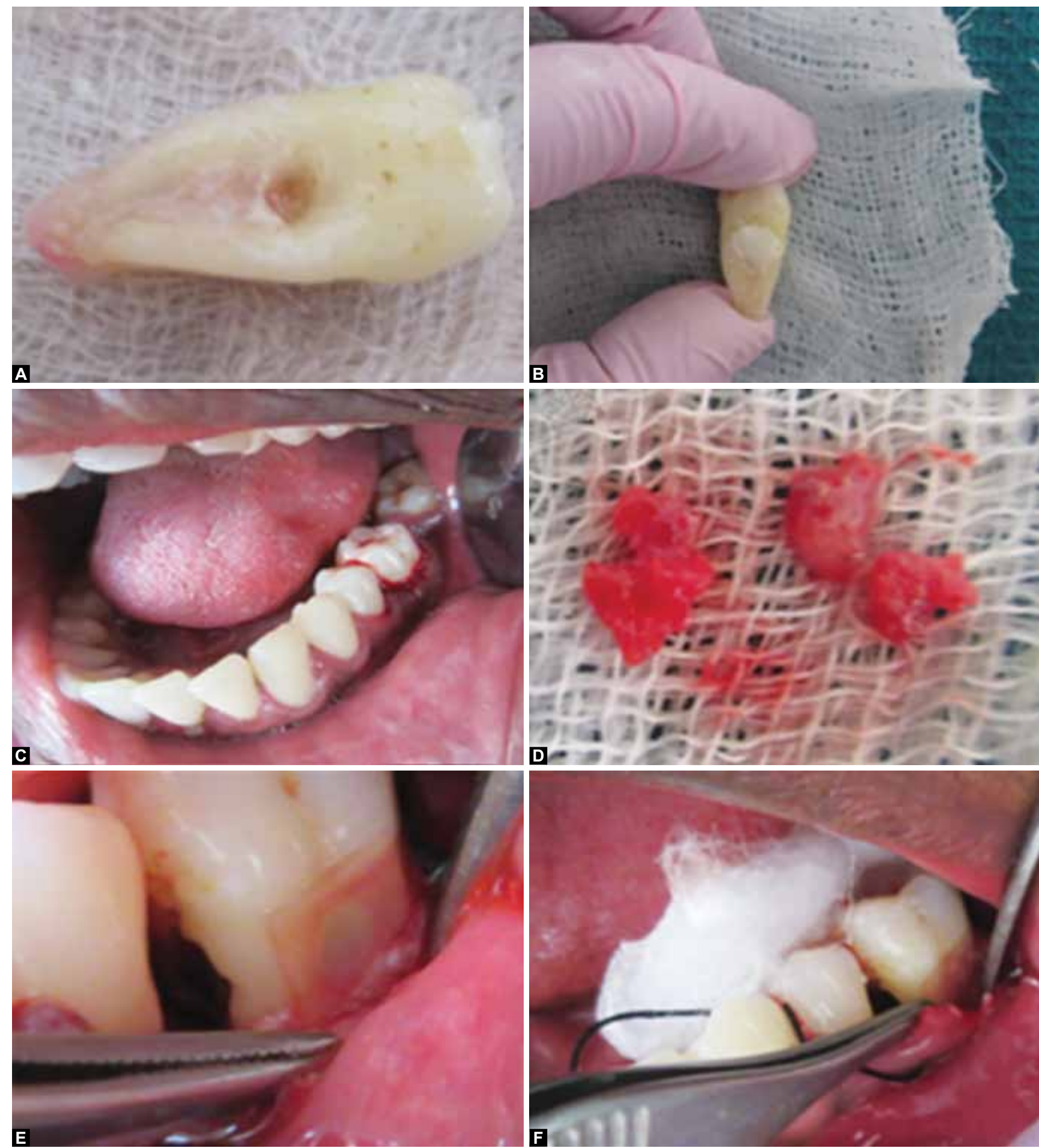

Figs $3 A$ to $F:(A)$ Curettage of the resorptive site, $(B)$ resorptive site repaired with biodentine, $(C)$ repositioning of the tooth into its socket, (D) curettage of the gingival tissues, (E) placement of GTR membrane and (F) placement of bone graft

the diagnosis of a case of cervical root resorption in maxillary central incisor with clinical and radiographic assessment. In our case, the suspected site of resorption is on the mesial surface of the mesial root of 36 which is clinically an inaccessible site. This justifies the cause for using $\mathrm{CBCT}$ as an additional diagnostic aid in our case. One of the definitive indications of CBCT in endodontic is for the diagnosis of cervical resorption. ${ }^{9,10}$ We used a limited field of view CBCT for confirming the presence of resorptive lesion.
In our case, 36 exhibited class 3 ECIR involving the mesial surface of the mesial root. This site is inaccessible to conventional intraoral surgical procedures. So, we planned to perform intentional reimplantation. The success rates of intentional reimplantation in molars ranges from 52 to $95 \% .^{6,11}$

One of the indications of intentional replantation is when a perforating internal or external resorption is present but surgery is impractical, ${ }^{12}$ which is typical of our case scenario. 


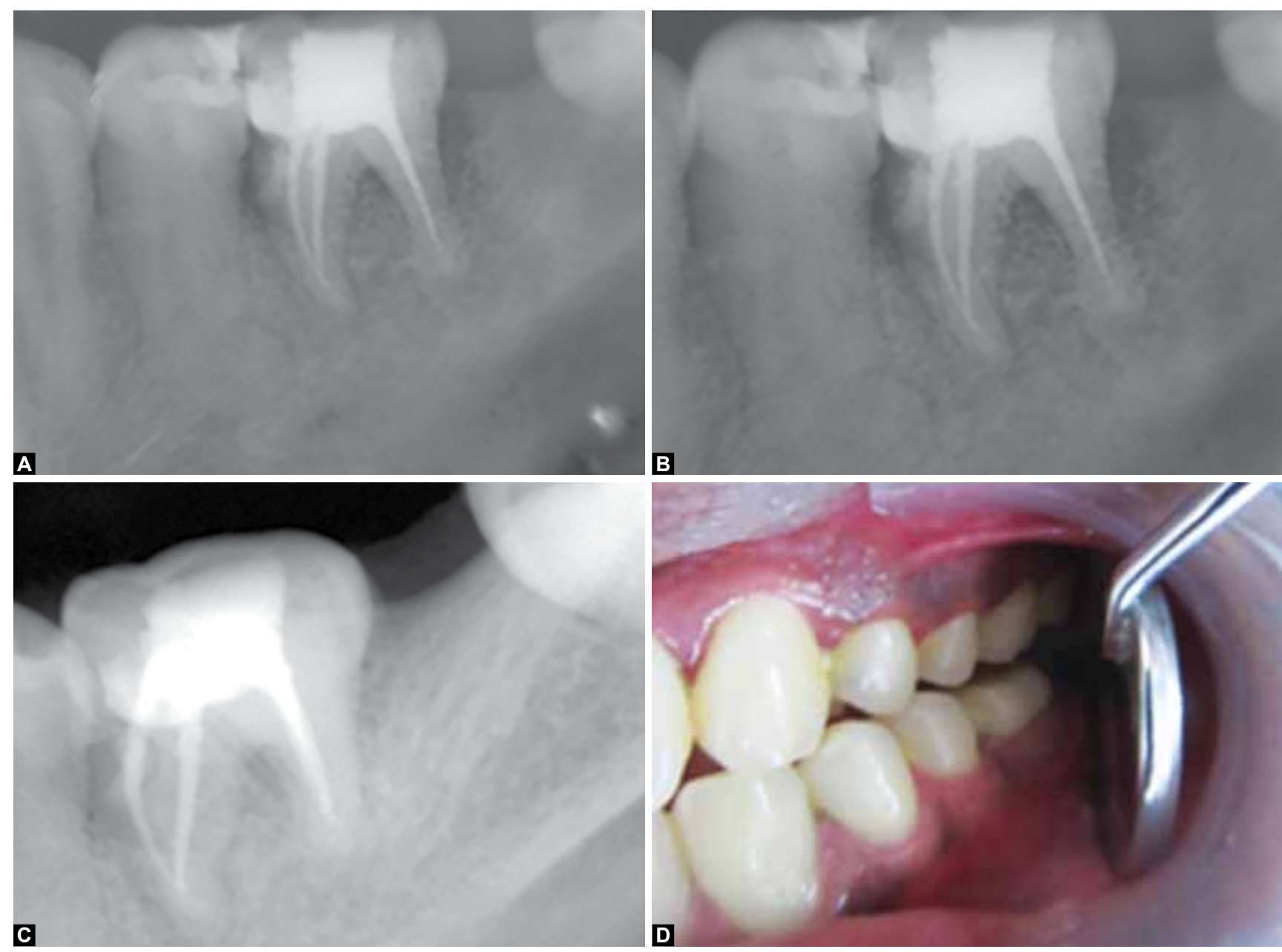

Figs 4A to D: (A) Immediate postoperative radiograph, (B) 3 months postoperative radiograph and (C and D) 10 months postoperative radiograph and clinical photograph

There are lot of factors which influence the outcome of intentional replantation positively: the extraoral time, contact of the root surface in normal saline, patient's age, root end filling material used, presence of preoperative radiolucency, minimal manipulation of the tooth surface and the kind of the tooth (single-rooted or multirooted). Majority of the above-mentioned factors was in favor of the proposed treatment plan.

Endodontic treatment was completed within 20 minutes and root end resection was done to eliminate the lateral canals in the apical third of the root. Endodontic treatment was opted to be performed extraorally because performing endodontic treatment intraorally prior to extraction might result in weakening of the coronal tooth structure and result in breakage of the tooth during extraction. Retrograde preparation was done and glass ionomer cement was used as the root end filling material. Glass ionomer cement is a biocompatible material which chemically adheres to the tooth surface and has good sealing ability. It possesses antibacterial properties ${ }^{13}$ and provides good epithelial and connective tissue adhesion with the adjacent structures and also possesses osteogenic potential. ${ }^{14}$
Biodentine was used to seal the resorption site. It is a novel tricalcium silicate-based material. It is biocompatible and enhances the hard-tissue formation in the vicinity by the release of alkaline phosphatase. Zhou et $1{ }^{15}$ has stated that Biodentine can be safely used in procedures requiring close approximation with the periodontal tissues.

Regeneration of the periodontal tissues was done with GTR membrane (resorbable collagen membrane) and bone regeneration was done with freeze-dried demineralized xenograft which is osteoconductive in nature.

After a follow-up period of 12 months, the patient was asymptomatic and radiographically exhibited no new resorptive lesion, periapical pathology or bone loss. A current systematic review by Torabinejad et $\mathrm{al}^{16}$ has stated that the success rate of intentionally replanted teeth is about $88 \%$. Thus with the current treatment protocol, there is predictable treatment outcome. Further long-term evaluation will be helpful in substantiating the treatment results.

\section{CONCLUSION}

External cervical root resorption is frequently encountered as an incidental diagnosis and the patients are usually 
asymptomatic. Prompt diagnosis and appropriate treatment plan will be very helpful in improving the longevity of such cases.

\section{ACKNOWLEDGMENT}

We would like to thank Dr K Malathi, MDS (Professor and Head of the Department of Periodontics) for immense support.

\section{REFERENCES}

1. Ne RF, Witherspoon DE, Gutmann JL. Tooth resorption. Quint Int 1999;30(1):9-25.

2. Andreasen JO. Luxation of permanent teeth due to trauma. A clinical and radiographic follow-up study of 189 injured teeth. Scand J Dent Res 1970;78(3):273-286.

3. Patel S, Ford TP. Is the resorption external or internal? Dental Update 2007;34(4):218-229.

4. Tronstad L. Root resorption-etiology, terminology and clinical manifestations. Endod Dent Traumatol 1988;4(6):241-252.

5. Heithersay GS. Invasive cervical resorption. Endod Topics 2004;7(1):73-92.

6. Grossmann LI. Intentional replantation of teeth. J Am Dent Assoc 1966 May;72(5):1111-1118.

7. Scarfe WC, Levin MD, Gane D, Farman AG. Review article use of cone beam computed tomography in endodontics. Int J Dent 2009;2009:1-20.
8. Ahmed N, Gopala Krishnan, Mony B, Parthasarthy H. External cervical resorption case report and a brief review of literature. J Nat Sci Biol Med 2014;5(1):210-214.

9. Cotton TP, Geisler TM, Holden DT, Schwartz SA, Schindler WG. Endodontic applications of cone-beam volumetric tomography. J Endod 2007;33(9):1121-1132.

10. Mota de Almeida FJ , Knutsson K, Flygare L. The impact of cone beam computed tomography on the choice of endodontic diagnosis. Int Endod J 2015;48(6):564-572.

11. Bender IB, Rossman LE. Intentional replantation of endodontically treated teeth. Oral Surg Oral Med Oral Pathol 1993;76(5):623-630.

12. Rouhani A, Javidi B, Habibi M, Jafarzadeh H. Intentional replantation: a procedure as a last resort. J Contemp Dent Prac 2011 Nov;12(6):486-492.

13. Vermeersch G, Leloup G, Delmée M, Vreven J. Antibacterial activity of glass-ionomer cements, compomers and resin composites: relationship between acidity and material setting phase. J Oral Rehabil 2005 May;32(5):368-374.

14. Sasanaluckit P, Albustany KR, Doherty PJ, Williams DF. Biocompatibility of glass ionomer cements. Biomaterials 1993 Oct;14(12):906-916.

15. Zhou HM, Shen $Y$, Wang ZJ, et al. In vitro cytotoxicity evaluation of a novel root repair material. J Endod 2013;39(4):478-483.

16. Torabinejad M, Dinsbach NA, Turman M, Handysides R, Bahjri K, White SN. Survival of intentionally replanted teeth and implant-supported single crowns: a systematic review. J Endod 2015 Jul;41(7):992-998. 